\title{
Regulation of S100A10 Gene Expression
}

\author{
Aleksandra Głowacka ${ }^{1}$, Paweł Bieganowski ${ }^{2} \mathbb{D}$, Ewelina Jurewicz ${ }^{1} \mathbb{D}$, Wiesława Leśniak ${ }^{1}$ (D), \\ Tomasz Wilanowski ${ }^{3, *(\mathbb{D})}$ and Anna Filipek $1, *$ (D)
}

1 Nencki Institute of Experimental Biology, Polish Academy of Sciences, 3 Pasteur Str., 02-093 Warsaw, Poland; al.glowacka@nencki.edu.pl (A.G.); e.jurewicz@nencki.edu.pl (E.J.); w.lesniak@nencki.edu.pl (W.L.)

2 Mossakowski Medical Research Institute, Polish Academy of Sciences, 5 Pawińskiego Str., 02-106 Warsaw, Poland; pawelb314@gmail.com

3 Institute of Genetics and Biotechnology, Faculty of Biology, University of Warsaw, 1 Miecznikowa Str., 02-096 Warsaw, Poland

* Correspondence: t.wilanowski@biol.uw.edu.pl (T.W.); a.filipek@nencki.edu.pl (A.F.); Tel.: +48-22-589-23-32 (A.F.); Fax: +48-22-822-53-42 (A.F.)

\begin{abstract}
S100A10, a member of the S100 family of $\mathrm{Ca}^{2+}$-binding proteins, is a widely distributed protein involved in many cellular and extracellular processes. The best recognized role of S100A10 is the regulation, via interaction with annexin A2, of plasminogen conversion to plasmin. Plasmin, together with other proteases, induces degradation of the extracellular matrix (ECM), which is an important step in tumor progression. Additionally, S100A10 interacts with 5-hydroxytryptamine 1B (5-HT1B) receptor, which influences neurotransmitter binding and, through that, depressive symptoms. Taking this into account, it is evident that S100A10 expression in the cell should be under strict control. In this work, we summarize available literature data concerning the physiological stimuli and transcription factors that influence S100A10 expression. We also present our original results showing for the first time regulation of $S 100 A 10$ expression by grainyhead-like 2 transcription factor (GRHL2). By applying in silico analysis, we have found two highly conserved GRHL2 binding sites in the 1st intron of the gene encoding S100A10 protein. Using chromatin immunoprecipitation (ChIP) and luciferase assays, we have shown that GRHL2 directly binds to these sites and that this DNA region can affect transcription of S100A10.
\end{abstract}

Keywords: cancer; gene expression; grainyhead-like 2; S100A10

Academic Editor: Donald Cameron

Received: 18 May 2021

Accepted: 28 June 2021

Published: 2 July 2021

Publisher's Note: MDPI stays neutral with regard to jurisdictional claims in published maps and institutional affiliations.

\section{Introduction}

The S100 protein family consists of over 20 low molecular weight (10-12 kDa), $\mathrm{Ca}^{2+}$. binding proteins expressed only in vertebrates [1]. In the human genome, genes encoding 17 members of the S100 family are clustered on chromosome 1 (1q21) [2]. Despite their shared localization, expression of S100 genes is not spatiotemporally synchronized and each gene has its own expression pattern [3,4]. S100 proteins bind $\mathrm{Ca}^{2+}$ through two "EF-hand" motifs. In a $\mathrm{Ca}^{2+}$-bound form, they interact with a variety of protein ligands which, in consequence, leads to regulation of numerous processes inside and outside the cell $[5,6]$.

S100A10 is a unique member of the S100 protein family in that it does not bind $\mathrm{Ca}^{2+}$ and is insensitive to changes in $\mathrm{Ca}^{2+}$ concentration. Both "EF hand" motifs in S100A10 have amino acid substitutions that allow them to maintain a conformation, which resembles that of other S100 proteins in a $\mathrm{Ca}^{2+}$-bound form. This means that S100A10 is permanently locked in an active state [7]. Inside the cell, S100A10 forms a heterotetrameric complex with annexin A2, a protein that binds $\mathrm{Ca}^{2+}$ and phospholipids [8]. The annexin A2-S100A10 complex is involved in many membrane-associated processes, e.g., trafficking, fusion, microdomain or lipid raft organization and cytoskeleton-membrane binding. Additionally, this complex binds F-actin, which suggests that it is involved in cytoskeletal reorganization and regulation. Moreover, the annexin A2-S100A10 complex interacts with plasminogen 
and facilitates its conversion into plasmin, a serine protease that is responsible for fibrinolysis but can also activate metalloproteinases and degrade extracellular matrix (ECM) proteins [9]. Dysregulation of plasminogen conversion to plasmin plays an important role in the etiology of many cancer and non-cancer diseases. It is well known that degradation of extracellular matrix proteins, together with inflammation, promotes tumor growth, invasiveness, and metastasis (Figure 1). It is thus not surprising that an elevated level of S100A10 is a common feature of many cancers, such as squamous cell carcinoma, colon, lung, breast, or pancreatic cancer [10-12]. The role of S100A10 in other processes such as modulation of serotonin and other receptors, regulation of ion channel level and activity, or involvement in macrophage migration has been extensively reviewed $[13,14]$. It is only natural that expression of S100A10 must be flexible enough to meet these functional demands. In this work, we present literature data concerning various factors that influence S100A10 expression and our original results that show that the gene is regulated by GRHL2.

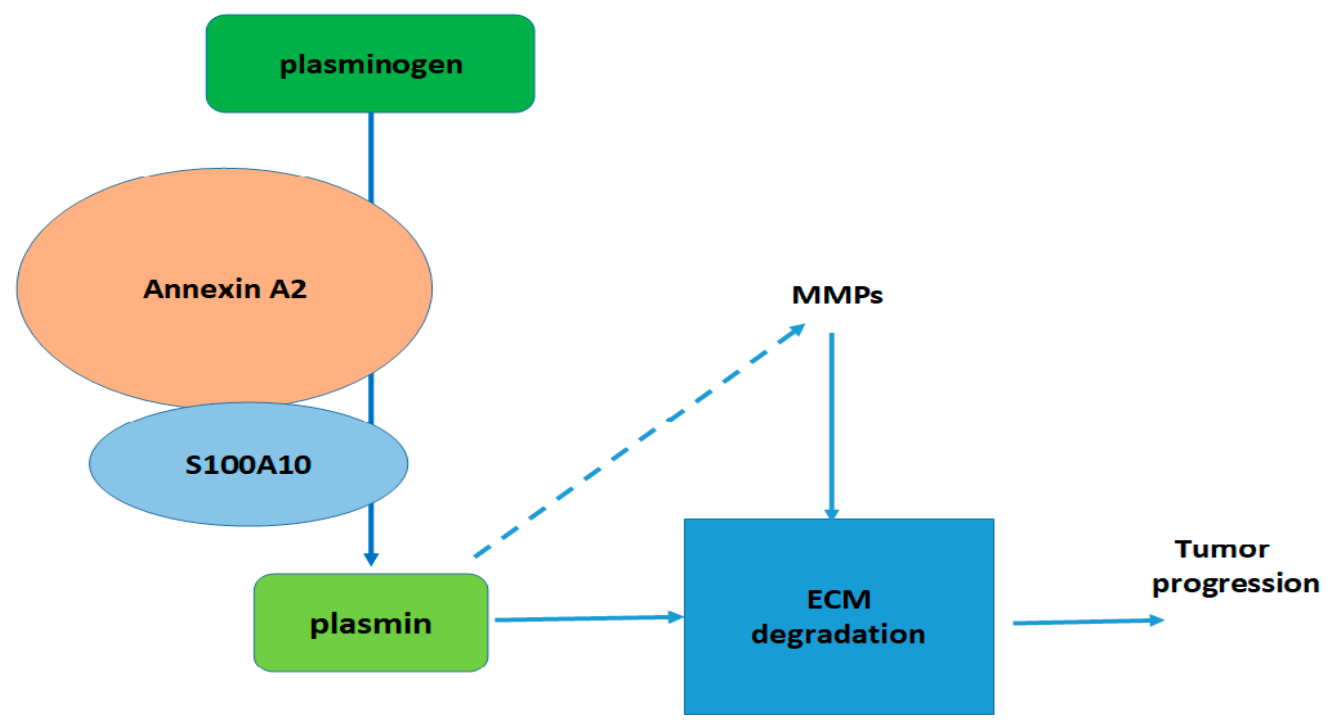

Figure 1. Role of S100A10 in tumor progression.

\section{Regulation of S100A10 Expression-Literature Data}

The gene encoding S100A10 is highly inducible and its expression seems to be regulated by numerous external and internal factors. Studies performed by different research groups identified many physiological stimuli that promoted S100A10 expression, among them transforming growth factor- $\beta$ (TGF- $\beta$ ), gonadotrophin (GD), epidermal growth factor (EGF), basic fibroblast growth factor (bFGF), interleukin $1 \beta$ (IL-1 $\beta$ ) [13], brain-derived neurotrophic factor (BDNF) [15], fibroblast growth factor 2 (FGF2) [16], and nitric oxide (NO) [17]. Theoretical analysis of the S100A10 promoter identified many potential regulatory sites, among them $\gamma \mathrm{IRE}, \mathrm{GAS}$, and GRE, and consensus binding sequences for the following transcription factors: AP-1, Sp1, Sp2, NFkB, HIF1, ATF, and CTF-NF1 [13]. Over time, the binding of these and other transcription factors to their cognate binding sites within the regulatory regions of S100A10 has been verified experimentally, providing relevant information on the regulation of S100A10 expression. It was proposed, for example, based on the effects of siRNA-induced knockdown of several transcription factors, that c-Fos and c-Jun may act as activators, while CREM, Fosl2, STAT3, Sp1, and SRF may act as inhibitors, of S100A10 expression [16]. Indeed, the binding of the c-Fos/c-Jun dimer, which forms the AP-1 transcription factor, to the 1st exon of S100A10, was confirmed by chromatin immunoprecipitation (ChIP) and its stimulatory effect on expression was shown in a luciferase assay. Activation of S100A10 transcription by AP-1 is mediated by MAPK, PI3K, and JNK cascades in response to BDNF or FGF2 stimulation and constitutes one of the mechanisms of antidepressant response in which S100A10 plays a significant role [16]. Another group demonstrated the binding of Sp1, and probably also of Sp3, to 
a GC-box within the -96 to -70 segment of the S100A10 promoter using electrophoretic mobility shift assay (EMSA) [18]. It was shown that Sp1 upregulates S100A10 expression in response to nitrosative stress induced in motor neuron-like cell line, NSC34, by high NO concentration. Increased S100A10 level impairs the function of TASK1, a two-pore domain $\mathrm{K}+$ channel, and contributes to degeneration of motor neurons, while silencing of S100A10 is neuroprotective [18].

The mechanism of the responsiveness of S100A10 to inflammatory cues has also been studied. It was shown that, in response to interferon $\gamma$ stimulation, STAT1 binds to two GAS sequences located at positions -1219 and -1090 of the gene promoter [19]. The effect of STAT1 binding on S100A10 expression was assessed by the luciferase assay, which demonstrated that overexpression of wild-type STAT1 in two epithelial cell lines, BEAS-2B and HeLa, increased luciferase activity, while a phosphorylation deficient (Tyr 701) STAT1 mutant had no effect.

Chedeville et al. [20] observed increased S100A10 expression following HIF1 $\alpha$ upregulation in glioblastoma cells and samples from glioblastoma patients as compared with normal tissue [20]. Accordingly, the binding of HIF1 $\alpha$ and HIF1 $\beta$, but not HIF2 $\alpha$, to the 1st exon of S100A10, 103 bp downstream of the transcription start site, was shown by ChIP. This binding occurred following hypoxia or after treatment of glioblastoma cells with anti-cancer drugs [21]. The involvement of HIF1 $\alpha$ links S100A10 expression with hypoxic conditions characteristic for many cancer tissues.

It was also revealed that dexametasone-induced S100A10 expression involves glucocorticoid receptor (GR) binding sites in the promoter sequence [22]. Three possible GRE sites $(-223$ to $-241,-354$ to -372 , and -427 to -445$)$ were identified in the S100A10 promoter region; at least two of them were found to be functional by means of mutational analysis. The binding of GR to these sites was shown by ChIP after dexametasone stimulation of neuroblastoma SH-SY5Y cells [22].

The stimuli and transcription factors known to be involved in regulation of S100A10 expression are listed in Table 1. Of note, in many cases, thanks to the research efforts described above, the whole signaling pathways, starting from the initial signal downstream to transcription factors that transduce the stimulatory cue into a higher transcription rate, have been revealed. Although most data concern a single activating stimulus and/or transcription factor, it is evident that in vivo these factors act in concert to modulate S100A10 transcription according to cell's needs.

Table 1. Stimuli and transcription factors which influence S100A10 expression.

\begin{tabular}{ccc}
\hline Stimulus & Transcription Factor & Reference \\
\hline Transforming growth factor $\beta$ (TGF $\beta)$ & ND & {$[13]$} \\
\hline Epidermal growth factor (EGF) & ND & {$[13]$} \\
\hline Interleukin $1 \beta($ IL-1 $\beta)$ & ND & {$[13]$} \\
\hline $\begin{array}{c}\text { Brain-derived neurotrophic factor (BDNF); } \\
\text { basic fibroblast growth factor 2 (FGF2) }\end{array}$ & c-Jun/c-Fos (AP-1) & {$[16]$} \\
\hline Nitric oxide (NO) & Sp1, Sp3 & {$[17,18]$} \\
\hline Gonadotrophin (GD) & ND & {$[13,22]$} \\
\hline Hypoxia, anti-cancer drugs & HIF1 & {$[13,20,21]$} \\
\hline Interferon $\gamma$ & STAT1 & {$[19,20]$} \\
\hline ND & NFkB & {$[13]$} \\
\hline ND & CTF-NF1 & {$[13]$} \\
\hline ND & GRHL2 & Present work \\
\hline
\end{tabular}

ND—not determined. 


\section{Regulation of S100A10 Expression by GRHL2 Transcription Factor-Original Data \\ 3.1. Influence of GRHL2 on S100A10 Expression}

GRHL2 is a member of the grainyhead-like transcription factor family [23]. GRHL2 regulates processes such as proliferation and differentiation [24] and thus its dysregulated expression is a common cause of malignant transformation. Interestingly, depending on the cancer type, the disease can arise due to either up- or downregulation of this transcription factor. This is because GRHL2 may act both as tumor suppressor, by inhibiting epithelial to mesenchymal transition, or as an oncogene, by inducing cell proliferation and hTERT expression. An increased level of GRHL2 was found in squamous cell carcinoma, gastric, liver, and colon cancer $[25,26]$ and is usually associated with bad patient prognosis [24]. Increased expression of the gene encoding GRHL2 and, as mentioned above, of that encoding S100A10 is common for malignant transformation. Examples include hepatocellular carcinoma, non-small-cell lung cancer, colorectal cancer, and pancreatic ductal adenocarcinoma $[11,26]$. Thus, it was interesting to check whether GRHL2 transcription factor is involved in regulation of the gene encoding S100A10 protein. To show this, we used in silico and experimental approaches described in detail in Supplementary File (Materials and Methods).

A theoretical analysis of putative GRHL2 transcription factor binding sites in the gene encoding human S100A10 protein (GENEBANK NC_000001.11 chr.1, GRCh38.p13 region complement 151982915-151994859) was performed using the MatInspector program (https:/ / www.genomatix.de/solutions/genomatix-software-suite.html (accessed on 2 July 2021)). This program identified five potential, but only two highly conserved, binding sites for GRHL2 located in the 1st intron of S100A10 (Figure 2A-GRHL2 No1 and GRHL2 No2 and Figure 2B-bold letters) [27]. These sites were only slightly divergent from the consensus $5^{\prime}$-AACCGGTT- $3^{\prime}$ sequence. No sequence that might bind GRHL2 was found in the promoter region.
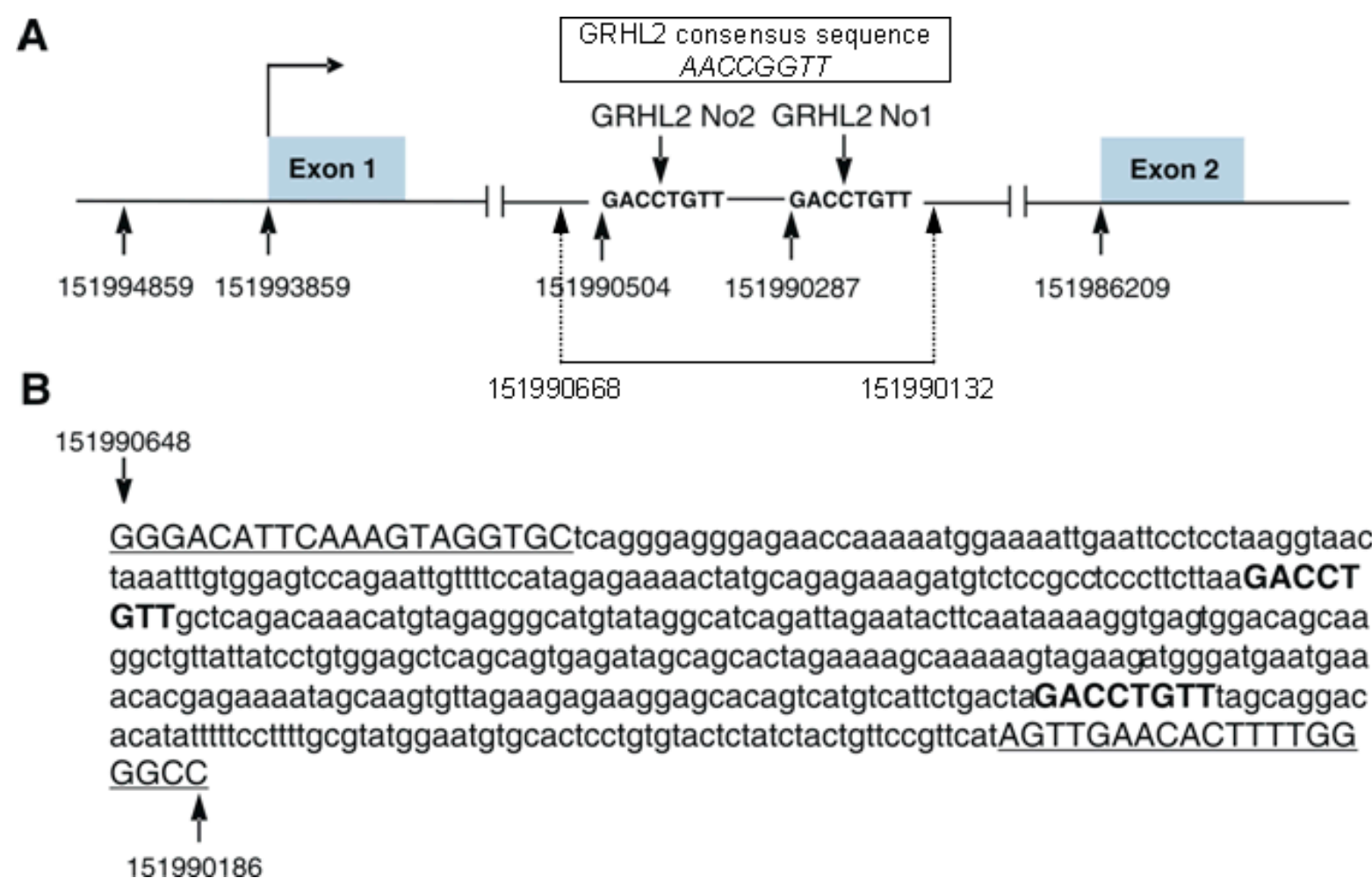

Figure 2. Schematic representation of the gene encoding S100A10 protein (A) and sequence of the intronic fragment amplified in ChIP (B). (A) Nucleotide sequence of two GRHL2 binding sites, GRHL2 No1 and GRHL2 No2. The 536 bp intronic fragment (151990668-151990132) cloned into pTA-Luc plasmid is indicated by dotted lines with arrowheads. (B) Nucleotide sequence of two GRHL2 binding sites (bold) and sequences covered by primers used for ChIP (underlined). Numbers are given according to GENEBANK NC_000001.11. 
To assess the influence of GRHL2 on S100A10 transcription, a luciferase assay was employed. For that, a reporter plasmid (pTA-Luc-S100A10-GRHL2-WT) containing the luciferase gene and a 536 bp long intronic sequence (chr1:151 990 668-151 990 132), comprising the identified GRHL2 binding sites, was constructed as described in Supplementary File (Material and Methods and Table S1) and used in a Dual-Luciferase Reporter Assay System. For that, HEK293 (human embryonic kidney) cells were transfected with the pRL-SV40 reference plasmid, as an internal control, and with the pTA-Luc-S100A10-GRHL2-WT containing the examined sequence in the 1st intron of the S100A10. Cells were co-transfected with plasmid encoding GRHL2 with a FLAG tag (EX-W2222-M12-GRHL2-3xFLAG) or control plasmid (EX-NEG-M12-3xFLAG). Luciferase activity was assessed after $24 \mathrm{~h}$ using a Glomax 20/20 luminometer. As shown in Figure 3A, overexpression of GRHL2 led to an increase in luciferase activity, which indicates that this transcription factor regulates S100A10 expression.

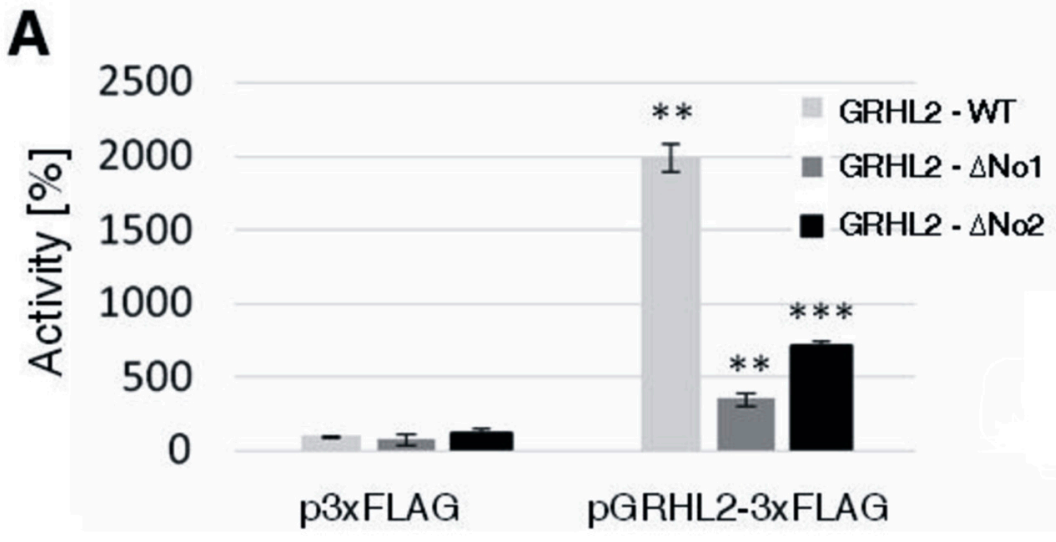

B

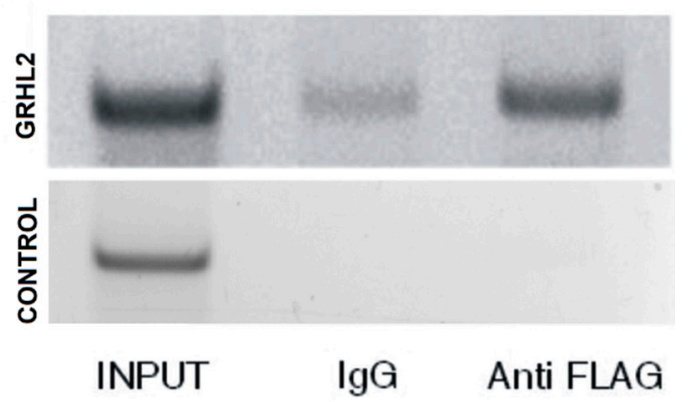

Figure 3. Influence of GRHL2 on S100A10 expression and its binding to the S100A10 intronic sequence. (A) Luciferase assay with plasmids containing the S100A10 intronic sequence wild-type (GRHL2-WT) or lacking one of the GRHL2 binding sites (GRHL2- $\Delta$ No1 or GRHL2- $\Delta$ No2). Statistical analysis of results obtained from $n=3$ experiments was performed with the use of Student's $t$-test. Results are presented as means \pm standard deviation. The level of statistical significance is indicated using ${ }^{* *} p \leq 0.01$ or ${ }^{* *} p \leq 0.001$. (B) A representative ChIP result-PCR products obtained with the use of primers encompassing both potential GRHL2 binding sites (upper panel) and primers that amplify a region of DNA not predicted to bind GRHL2 (lower panel).

To confirm these results, each potential binding site for the GRHL2 transcription factor was subsequently deleted using pTA-Luc-S100A10-GRHL2-WT as a template in a PCR reaction with appropriate primers (Supplementary File, Table S1). The resulting products were named pTA-Luc-S100A10-GRHL2- $\Delta$ No1 and pTA-Luc-S100A10-GRHL2- $\Delta$ No2. The luciferase assay was performed as described above. As shown in Figure $3 \mathrm{~A}$, overexpression of GRHL2 in HEK293 cells transfected with plasmids containing the examined sequence lacking one of the GRHL2 binding sites led to a statistically significant decrease in luciferase activity.

To further assess the functionality of GRHL2 transcription binding sites in the 1st intron of S100A10, a ChIP assay was performed. For that HEK293 cells were transfected with plasmid EX-W2222-M12-GRHL2-3xFLAG and, $24 \mathrm{~h}$ later, were fixed with 1\% formaldehyde, lysed, and sonicated into about $500 \mathrm{bp}$ long fragments (Materials and Methods and Figure S1 in the Supplementary File). Overexpression of FLAG-tagged GRHL2 in the ChIP assay was necessary since available antibodies against GRHL2 are not specific and may recognize other transcription factors of this family. The lysate was incubated overnight with mouse IgG (control) or mouse monoclonal anti-FLAG antibody and the DNA-protein complexes were immunoprecipitated using Protein A/G Agarose. The immunoprecipitated DNA was used as a template for PCR reactions with primers flanking the two potential 
GRHL2 binding sites or primers flanking the region not predicted to bind GRHL2 (control) (Supplementary File, Table S1).

In accordance with an in silico analysis, which ranked the two GRHL2 sites as 22 and 37 top hits out of more than 255 hits (0.8 threshold) (Supplementary File, Table S2), a clear PCR product of an appropriate length was detected only in the sample containing the template immunoprecipitated with antibody against the FLAG tag and amplified with primers encompassing the two GRHL2 binding sites (Figure 3B, upper panel). No PCR product was visible in a control experiment (Figure 3B, lower panel). Thus, the results indicate that the GRHL2 transcription factor binds to its predicted sites within the 1st intron of S100A10 and therefore may directly regulate expression of this gene.

\subsection{Analysis of S100A10 mRNA in RC-124 Cells with Silenced GRHL2 Expression}

To check the effect of the GRHL2 transcription factor on S100A10 expression, we used kidney epithelial RC-124 cells with diminished level of this transcription factor (transfected with GRHL2 shRNA) [28]. The level of GRHL2 mRNA in these cells and in control ones (transfected with scrambled shRNA) was analyzed using RT-qPCR. The TaqMan Fast Universal Master Mix with TaqMan Probes (ID: Hs02800695_m1 for HPRT and Hs00227745_m1 for GRHL2) was used and the reaction was performed in a 7900HT Fast Real-Time PCR System.

The obtained results were analyzed using the comparative $\Delta \Delta C_{\mathrm{t}}$ method and gene expression was normalized to the HPRT1 housekeeping gene. As can be seen in Figure 4A, indeed, the level of GRHL2 mRNA in RC-124 with silenced GRHL2 expression was diminished when compared to control cells.
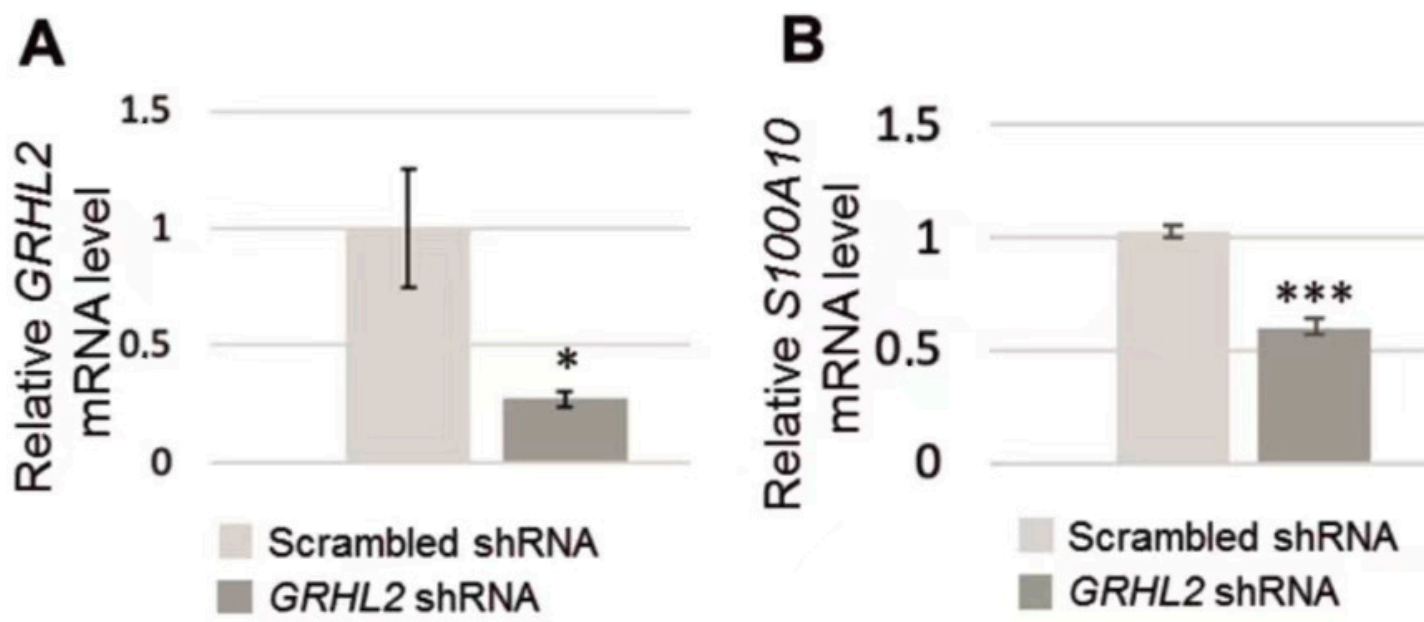

Figure 4. S100A10 mRNA in RC-124 cells with silenced GRHL2. (A,B) RT-qPCR analysis showing the level of GRHL2 mRNA and S100A10 mRNA, respectively. (A,B): statistical analysis of results obtained from $n=3$ experiments was performed with the use of Student's $t$-test. Results are presented as means \pm standard deviation. The level of statistical significance is indicated using ${ }^{*} p \leq 0.05$ or ${ }^{* * *} p \leq 0.001$. Scrambled shRNA represents control cells while GRHL2 shRNA—cells with silenced GRHL2 expression.

S100A10 mRNA level was then analyzed by RT-qPCR using the SYBRGreen system and appropriate primers (Supplementary File, Table S1). As it can be seen in Figure 4B, the level of S100A10 mRNA in RC-124 cells with silenced GRHL2 expression is lower than in control ones. In both cases, the differences were statistically significant.

In summary, by applying in silico analysis, we identified two highly conserved GRHL2 transcription factor binding sites in the 1st intron of the gene encoding the S100A10 protein. Such intronic location of the binding sites conforms to the results of ChIP-seq analyses, which show that GRHL2 binding occurs more frequently within intragenic and intronic regions than gene promoters $[29,30]$. Intronic GRHL2 binding sites were identified in 
CDH1 (encoding E-catherin) and other genes [30,31]. The presence of regulatory sequences outside the promoter region is not uncommon [32]. For instance, it was found that $32-40 \%$ of transcription binding sites for HSF are located in introns [33]. Additionally, functional binding sequences for NFAT1 have been identified outside the gene promoter [34]. By performing chromatin immunoprecipitation (ChIP) and luciferase activity assays, we have found that GRHL2 directly binds to the intronic binding sites and that it is able to stimulate transcription of S100A10. Moreover, RT-qPCR showed that in cells with silenced GRHL2 expression, S100A10 mRNA level was lower than in control cells. Altogether, in this work, we show for the first time that GRHL2 transcription factor regulates expression of S100A10. Since both proteins are involved in proliferation/tumorigenesis/metastasis, their interrelated regulation might have some impact on cancer development and progression.

\section{Conclusions}

S100A10 is a member of the S100 $\mathrm{Ca}^{2+}$-binding protein family. S100A10 is involved in regulation of various processes, among them in plasmin production and regulation of the level of matrix metalloproteinases. Thus, in consequence, S100A10 has an effect on degradation of the extracellular matrix (ECM), which is an important step in tumorigenesis/metastasis.

Up to now, several reports have been published concerning regulation of S100A10 expression. Based on all these data, it is evident that it can be influenced by many different stimuli, which, together with a range of transcription factors, ensure that the S100A10 protein level meets the demands of a given cell. Our work has identified yet another transcription factor, that is GRHL2, a protein which, among others, plays a role in tumorigenesis. Expression of GRHL2 is increased in many cancers [26] and the same applies to S100A10 [9]. Cancer types in which the expression of both GRHL2 and S100A10 is simultaneously increased include hepatocellular carcinoma, non-small-cell lung cancer, colorectal cancer, and pancreatic ductal adenocarcinoma $[9,26]$. In the above examples, the mechanism of GRHL2 action does not necessarily involve epithelial-mesenchymal transition. Taking into account the increased level of S100A10 and GRHL2 in many cancers, it is possible that regulation of S100A10 expression by GRHL2 might have an impact on cancer progression and metastasis.

Supplementary Materials: The following are available online at https:/ /www.mdpi.com/article/10 .3390 /biom11070974/s1, Materials and methods, Figure S1: Agarose gel showing the approximate length of the sheared chromatin used in ChIP, Table S1: Sequences of primers, Table S2: Putative transcription binding sites in S100A10 according to MatInspector.

Author Contributions: Each author participated sufficiently in the work to take public responsibility for the appropriate portions of the article content. Authors who made substantial contributions to: Conceptualization, A.F.; T.W.; W.L.; A.G.; P.B. Methodology, A.G.; P.B.; E.J.; W.L.; A.F.; T.W. Formal Analysis, A.G.; P.B.; E.J.; W.L.; A.F.; T.W. Investigation, A.G.; E.J.; P.B.; T.W. Resources, T.W.; A.F. Data Curation, A.G.; P.B.; T.W. Writing-Original Draft Preparation, A.G.; A.F.; W.L. Writing-Review and Editing, A.F.; A.G.; W.L.; P.B.; T.W.; E.J. Visualization, A.G.; E.J. Supervision, A.F.; T.W. Project Administration, A.F. Funding Acquisition, A.F. All authors have read and agreed to the published version of the manuscript.

Funding: This work was supported by grants from the National Science Centre 2018/29/B/NZ4/01384 to A.F. and 2016/21/B/NZ1/00279 to T.W., and by statutory funds from the Nencki Institute.

Acknowledgments: Authors are grateful to M. Dabrowski for help with bioinformatics analysis.

Conflicts of Interest: The authors declare no conflict of interest. 


$\begin{array}{ll}\text { Abbreviations } \\ \text { BDNF } & \text { brain-derived neurotrophic factor } \\ \text { BSA } & \text { bovine serum albumin } \\ \text { ChIP } & \text { chromatin immunoprecipitation assay } \\ \text { DTT } & \text { dithiothreitol } \\ \text { EDTA } & \text { ethylenediaminetetraacetic acid } \\ \text { EGF } & \text { epidermal growth factor } \\ \text { FGF2 } & \text { fibroblast growth factor } 2 \\ \text { bFGF } & \text { basic fibroblast growth factor } \\ \text { GAS } & \text { interferon } \gamma \text { activation site } \\ \text { GD } & \text { gonadotrophin } \\ \text { GR } & \text { glucocorticoid receptor } \\ \text { GRE } & \text { glucocorticoid response element } \\ \text { GRHL2 } & \text { grainyhead-like } 2 \\ \text { hTERT } & \text { human telomerase reverse transcriptase } \\ \text { JNK } & \text { c-Jun N-terminal kinase } \\ \text { L-1 } \beta & \text { interleukin } 1 \beta \\ \text { IP } 3 & 1,4,5 \text {-trisphosphate inositol } \\ \gamma \text { IRE } & \text { interferon } \gamma \text { response element } \\ \text { MAP } & \text { mitogen activated protein } \\ \text { TGF- } \beta & \text { transforming growth factor- } \beta\end{array}$

\section{References}

1. Gonzalez, L.L.; Garrie, K.; Turner, M.D. Role of S100 proteins in health and disease. Biochim. Biophys. Acta 2020, $1867,118677$. [CrossRef]

2. Schäfer, B.W.; Wicki, R.; Engelkamp, D.; Mattei, M.G.; Heizmann, C.W. Isolation of a YAC clone covering a cluster of nine S100 genes on human chromosome 1q21: Rationale for a new nomenclature of the S100 calcium-binding protein family. Genomics 1995, 25, 638-643. [CrossRef]

3. Marenholz, I.; Heizmann, C.W.; Fritz, G. S100 proteins in mouse and man: From evolution to function and pathology (including an update of the nomenclature). Biochem. Biophys. Res. Commun. 2004, 322, 1111-1122. [CrossRef] [PubMed]

4. Leśniak, W. Epigenetic regulation of S100 protein expression. Clin. Epigenet. 2011, 2, 77-83. [CrossRef]

5. Gross, S.R.; Sin, C.G.; Barraclough, R.; Rudland, P.S. Joining S100 proteins and migration: For better or for worse, in sickness and in health. Cell Mol. Life Sci. 2014, 71, 1551-1579. [CrossRef]

6. Heizmann, C.W. Ca ${ }^{2+}$-Binding Proteins of the EF-Hand Superfamily: Diagnostic and Prognostic Biomarkers and Novel Therapeutic Targets. Methods Mol. Biol. 2019, 1929, 157-186.

7. Rescher, U.; Gerke, V. S100A10/p11: Family, friends and functions. Pflug. Arch. 2008, 455, 575-582. [CrossRef]

8. Bharadwaj, A.; Bydoun, M.; Hollow, R.; Waisman, D. Annexin A2 heterotetramer: Structure and function. Int. J. Mol. Sci. 2013, 14, 6259-6305. [CrossRef] [PubMed]

9. Li, C.; Ma, Y.; Fei, F.; Zheng, M.; Li, Z.; Zhao, Q.; Du, J.; Liu, K.; Lu, R.; Zhang, S. Critical role and its underlying molecular events of the plasminogen receptor, S100A10 in malignant tumor and non-tumor diseases. J. Cancer 2020, 11, 826-836. [CrossRef]

10. Sato, K.; Saiki, Y.; Arai, K.; Ishizawa, K.; Fukushige, S.; Aoki, K.; Abe, J.; Takahashi, S.; Sato, I.; Sakurada, A.; et al. S100A10 upregulation associates with poor prognosis in lung squamous cell carcinoma. Biochem. Biophys. Res. Commun. 2018, 505, 466-470. [CrossRef] [PubMed]

11. Tantyo, N.A.; Karyadi, A.S.; Rasman, S.Z.; Salim, M.R.G.; Devina, A.; Sumarpo, A. The prognostic value of S100A10 expression in cancer. Oncol. Lett. 2019, 17, 1417-1424. [CrossRef]

12. Saiki, Y.; Ho, A. Multiple functions of S100A10, an important cancer promoter. Pathol. Int. 2019, 69, 629-636. [CrossRef]

13. Madureira, P.; O'Connell, P.A.; Surette, A.P.; Miller, V.A.; Waisman, D.M. The biochemistry and regulation of S100A10: A multifunctional plasminogen receptor involved in oncogenesis. J. Biomed. Biotechnol. 2012, 2012, 353687. [CrossRef]

14. Seo, J.S.; Svenningsson, P. Modulation of Ion Channels and Receptors by p11 (S100A10). Trends Pharmacol. Sci. 2020, 41, 487-497. [CrossRef] [PubMed]

15. Warner-Schmidt, J.L.; Chen, E.Y.; Zhang, X.; Marshall, J.J.; Morozov, A.; Svenningsson, P.; Greengard, P. A role for p11 in the antidepressant action of brain-derived neurotrophic factor. Biol. Psychiatry 2010, 68, 528-535. [CrossRef] [PubMed]

16. Chottekalapanda, R.U.; Kalik, S.; Gresack, J.; Ayala, A.; Gao, M.; Wang, W.; Meller, S.; Aly, A.; Schaefer, A.; Greengard, P. AP-1 controls the p11-dependent antidepressant response. Mol. Psychiatry 2020, 25, 1364-1381. [CrossRef] [PubMed]

17. Pawliczak, R.; Cowan, M.J.; Huang, X.; Nanavaty, U.B.; Alsaaty, S.; Logun, C.; Shelhamer, J.H. p11 expression in human bronchial epithelial cells is increased by nitric oxide in a cGMP-dependent pathway involving protein kinase G activation. J. Biol. Chem. 2001, 276, 44613-44621. [CrossRef] 
18. García-Morales, V.; Rodríguez-Bey, G.; Gómez-Pérez, L.; Domínguez-Vías, G.; González-Forero, D.; Portillo, F.; Campos-Caro, A.; Gento-Caro, Á.; Issaoui, N.; Soler, R.M.; et al. Sp1-regulated expression of p11 contributes to motor neuron degeneration by membrane insertion of TASK1. Nat. Commun. 2019, 10, 3784. [CrossRef]

19. Huang, X.L.; Pawliczak, R.; Yao, X.L.; Cowan, M.J.; Gladwin, M.T.; Walter, M.J.; Holtzman, M.J.; Madara, P.; Logun, C.; Shelhamer, J.H. Interferon-gamma induces $\mathrm{p} 11$ gene and protein expression in human epithelial cells through interferon-gamma-activated sequences in the p11 promoter. J. Biol. Chem. 2003, 278, 9298-9308. [CrossRef] [PubMed]

20. Chédeville, A.L.; Lourdusamy, A.; Monteiro, A.R.; Hill, R.; Madureira, P.A. Investigating Glioblastoma Response to Hypoxia. Biomedicines 2020, 8, 310. [CrossRef]

21. Lu, H.; Xie, Y.; Tran, L.; Lan, J.; Yang, Y.; Murugan, N.L.; Wang, R.; Wang, Y.J.; Semenza, G.L. Chemotherapy-induced S100A10 recruits KDM6A to facilitate OCT4-mediated breast cancer stemness. J. Clin. Investig. 2020, 130, 4607-4623. [CrossRef]

22. Zhang, L.; Li, H.; Su, T.P.; Barker, J.L.; Maric, D.; Fullerton, C.S.; Webster, M.J.; Hough, C.J.; Li, X.X. Traumatic Stress Brain Study Group, Ursano R. p11 is up-regulated in the forebrain of stressed rats by glucocorticoid acting via two specific glucocorticoid response elements in the p11 promoter. Neuroscience 2008, 153, 1126-1134. [CrossRef] [PubMed]

23. Auden, A.; Caddy, J.; Wilanowski, T.; Ting, S.B.; Cunningham, J.M.; Jane, S.M. Spatial and temporal expression of the Grainyheadlike transcription factor family during murine development. Gene Exp. Patterns 2006, 6, 964-970. [CrossRef]

24. He, J.; Feng, C.; Zhu, H.; Wu, S.; Jin, P.; Xu, T. Grainyhead-like 2 as a double-edged sword in development and cancer. Am. J. Transl. Res. 2020, 12, 310-331.

25. Mlacki, M.; Kikulska, A.; Krzywinska, E.; Pawlak, M.; Wilanowski, T. Recent discoveries concerning the involvement of transcription factors from the Grainyhead-like family in cancer. Exp. Biol. Med. 2015, 1, 1-6. [CrossRef]

26. Kotarba, G.; Taracha-Wisniewska, A.; Wilanowski, T. Grainyhead-like transcription factors in cancer-Focus on recent developments. Exp. Biol. Med. 2020, 245, 402-410. [CrossRef]

27. Rifat, Y.; Parekh, V.; Wilanowski, T.; Hislop, N.R.; Auden, A.; Ting, S.B.; Cunningham, J.M.; Jane, S.M. Regional neural tube closure defined by the Grainy head-like transcription factors. Dev. Biol. 2010, 345, 237-245. [CrossRef] [PubMed]

28. Pawlak, M.; Kikulska, A.; Wrzesinski, T.; Rausch, T.; Kwias, Z.; Wilczynski, B.; Benes, V.; Wesoly, J.; Wilanowski, T. Potential protective role of Grainyhead-like genes in the development of clear cell renal cell carcinoma. Mol. Carcinog. 2017, 56, $2414-2423$. [CrossRef] [PubMed]

29. Wang, Z.; Wu, H.; Daxinger, L.; Danen, E.H.J. Genome-wide identification of binding sites of GRHL2 in luminal-like and basal A subtypes of breast cancer. BioRxiv 2020.

30. Chung, V.Y.; Tan, T.Z.; Tan, M.; Wong, M.K.; Kuay, K.T.; Yang, Z.; Ye, J.; Muller, J.; Koh, C.M.; Guccione, E.; et al. GRHL2-miR-200ZEB1 maintains the epithelial status of ovarian cancer through transcriptional regulation and histone modification. Sci. Rep. 2016, 18, 19943. [CrossRef]

31. Werth, M.; Walentin, K.; Aue, A.; Schönheit, J.; Wuebken, A.; Pode-Shakked, N.; Vilianovitch, L.; Erdmann, B.; Dekel, B.; Bader, M.; et al. The transcription factor grainyhead-like 2 regulates the molecular composition of the epithelial apical junctional complex. Development 2010, 137, 3835-3845. [CrossRef]

32. Rose, A.B. Introns as Gene Regulators: A Brick on the Accelerator. Front. Genet. 2019, 9, 672. [CrossRef] [PubMed]

33. Korfanty, J.; Stokowy, T.; Widlak, P.; Gogler-Piglowska, A.; Handschuh, L.; Podkowiński, J.; Vydra, N.; Naumowicz, A.; TomaJonik, A.; Widlak, W. Crosstalk between HSF1 and HSF2 during the heat shock response in mouse testes. Int. J. Biochem. Cell Biol. 2014, 57, 76-83. [CrossRef] [PubMed]

34. Kądziołka, B.; Leśniak, W.; Filipek, A. Regulation of CacyBP/SIP expression by NFAT1 transcription factor. Immunobiology 2017, 222, 872-877. [CrossRef] [PubMed] 